\title{
Diferenças de mensuração de acuidade visual e velocidade de leitura para perto entre pacientes com retinopatia diabética. Repercussão entre conceitos de deficiência visual parcial e cegueira legal
}

\author{
Differences in measurement of visual acuity and reading speed for near vision among patients \\ with diabetic retinopathy. Repercussion in concepts of partial vision loss and legal blindness

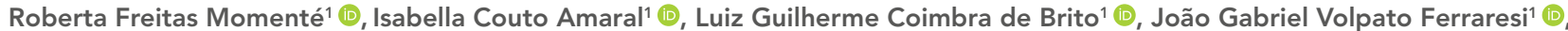 \\ Maria Luisa Gois da Fonsêca' ${ }^{1}$, Nadyr Antônia Damasceno² (D), Luiz Claudio Santos de Souza Lima' ${ }^{1}$, \\ Mauricio Bastos Pereira' ${ }^{1}$, Eduardo de França Damasceno ${ }^{1}$ ()

Descritores:

Acuidade visual; Cegueira legal; Leitura; Técnicas de diagnóstico oftalmológico

Keywords:

Visual acuity; Legal blindness; Reading; Ophthalmological diagnostic techniques

Submetido: 13/12/2020

Aceito: $8 / 6 / 2021$

Autor correspondente: Eduardo de França Damasceno Faculdade de Medicina Avenida Marques do Paraná, 303 - Centro CEP. 24210-350 - Niterói, RJ, Brasil E-mail: e_damasceno@yahoo.com

Observação: projeto idealizado como base didática do Programa de Iniciação Científica do Curso da Universidade Federal Fluminense e laureado com o prêmio de melhor trabalho científico no IX Congresso da Sociedade Brasileira de Visão Subnormal, em São Paulo, em 20 de junho 2019.

nstituição de realização do trabalho: Faculdade de Medicina, Universidade Federal Fluminense, Niterói, J, Brasil.

Fonte de auxílio à pesquisa: não financiado.

Conflitos de interesse: os autores declaram que não há conflitos de interesses.

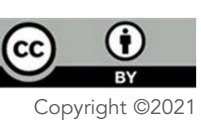

\section{RESUMO}

Objetivo: Comparar a acurácia entre a acuidade visual com melhor correção óptica em pacientes com retinopatia diabética e acuidade visual com correção óptica menor ou igual a 20/200 em ambos os olhos mensurada pela tabela de Snellen e pela tabela ETDRS, além da avaliação do tempo de leitura com tabela de leitura de Jaeger.

Métodos: Estudo observacional e transversal. ormação de Grupos de Pesquisa: o Grupo I foi formado por pacientes com acuidade visual de 20/200 e o Grupo II, por pacientes com acuidade visual maior de 20/200. Os pacientes de ambos os grupos foram subdivididos em aqueles com retinopatia diabética não proliferativa e os com retinopatia diabética proliferativa. As variáveis pesquisadas foram sexo, idade, tabela ETDRS nos subgrupos (igual a 20/200, igual a 20/400 e maior de 20/200 e 20/400) e tempo de leitura da tabela de Jaeger para perto. $O$ índice de significância estatística deste estudo foi de 5\%.

Resultados: Participaram do estudo 62 pacientes diabéticos, sendo 33 com retinopatia diabética não proliferativa e 29 com retinopatia diabética proliferativa. Dentre os pacientes com retinopatia diabética não proliferativa, 19 tinham acuidade visual com melhor correção óptica de 20/200 (30,6\% da amostra), e 13 pacientes eram portadores de retinopatia diabética proliferativa com acuidade visual com melhor correção óptica de 20/200 (20,9\% da amostra). A correlação entre a tabela Snellen e a tabela ETDRS revelou que 16 pacientes (25,8\% da amostra) com cegueira legal apresentaram acuidade visual entre 20/180 e 20/120 segundo a tabela ETDRS. Para os pacientes portadores de retinopatia diabética não proliferativa com acuidade visual de 20/200 pela tabela ETDRS, houve tempo médio de leitura para perto da tabela de Jaeger de 23,6 $\pm 1,4$ segundos. Na análise estatística da acuidade visual mensurada com a Tabela de Snellen em comparação com a Tabela de Leitura para perto (Jaeger), mensurando tempo de leitura, ahouve significância estatística $(p=0,04)$.

Conclusão: Pacientes diabéticos com cegueira legal segundo a tabela de Snellen apresentam sua rea capacidade visual residual subavaliada. É importante levar em consideração o tempo de leitura e o uso da tabela ETDRS para melhor acurácia.

\section{ABSTRACT}

Purpose: To compare the accuracy of best-corrected visual acuity in patients with diabetic retinopathy to corrected visual acuity $\leq 20 / 200$ in both eyes, measured by Snellen and ETDRS charts, in addition to evaluation of reading time by Jaeger chart.

Methods: An observational and cross-sectional study. Study Groups: Group I, patients with visual acuity 20/200, and Group II, patients with visual acuity $>20 / 200$. Patients were subdivided into those with nonproliferative diabetic retinopathy and with proliferative diabetic retinopathy. The study variables were sex, age, ETDRS chart in the subgroups (equal to 20/200; equal to 20/400 and greater than 20/200 and 20/400), and reading time by Jaeger chart on the near vision. The statistical significance was set at $5 \%$.

Results: A total of 62 diabetic patients participated in the study; in that, 33 with non-proliferative diabetic retinopathy and 29 with proliferative diabetic retinopathy. Among patients with non-proliferative diabetic retinopathy, 19 had best-corrected visual acuity 20/200 (30.6\% of sample), and 13 patients had proliferative diabetic retinopathy with best-corrected visual acuity $20 / 200$ (20.9\% of sample). The correlation between the Snellen and ETDRS charts revealed 16 patients (25.8\% of sample) with legal blindness presented visual acuity between 20/180 and 20/120, as per the ETDRS chart. For patients with non-proliferative diabetic retinopathy with visual acuity 20/200 measured by the ETDRS chart, the mean reading time to near vision was $23.6 \pm 1.4$ seconds, as measured by Jaeger chart. In the statistical analysis, the comparison of visual acuity measured by Snellen chart, with the reading chart to near vision (Jaeger) measuring reading time, was statistically significant ( $p=0.04)$.

Conclusion: Diabetic patients with legal blindness as per Snellen chart showed their real underestimated residual visual capacity. It is important to consider reading time and use of the ETDRS chart for better accuracy. 


\section{INTRODUÇÃO}

A mensuração da acuidade visual (AV) é o exame oftalmológico básico mais comum numa avaliação ocular, sendo baseado em optotipos ou em um conjunto de gráficos com letras ou desenhos de alto contraste e arco de um grau de ângulo visual. ${ }^{(1-3)} \mathrm{A}$ estimativa da AV é comumente aferida por meio da tabela de Snellen, sendo uma ferramenta fundamental da consulta médica oftalmológica. (4,5) Esta avaliação assume suma importância para caracterizar pacientes com deficiência visual.

Dentre as causas de baixa $A V$, a retinopatia diabética (RD) tem sido apontada como morbidade ocular de grande importância na etiologia da baixa AV permanente mundial. ${ }^{(6-8)}$ Essa etiologia foi escolhida para este estudo devido à sua variabilidade de comprometimento macular, quer por sua condição edematosa e de variável resposta terapêutica antiangiogênica atual, quer também por sua condição de complicação de doença crônica, o diabetes mellitus, com impacto visual, principalmente em sua forma de RD proliferativa. ${ }^{(9,10)}$

A estimativa da deficiência de visão pela medida de AV tem sido baseada em critérios de cegueira legal, conforme apregoado pelo Ministério da Saúde: AV menor do que 20/200 com correção óptica pela tabela de Snellen, no olho de melhor visão. ${ }^{(11,12)}$

Entretanto, uma hipótese de subestimação da capacidade visual por superestimação do conceito de deficiência visual poderia acontecer, uma vez que esse tipo de acontecimento se constitui numa das limitações da tabela de Snellen, projetada com optotipos sem efeito bidimensional, em perspectiva de linha a linha, de espaços variados e não padronizados entre cada optotipo, bem como portadora de um número variável de letras a cada linha de nível da AV. ${ }^{(13,14)}$ Assim, caso se comparassem pacientes com diagnóstico de deficiência visual pela aplicação de outros tipos de testes psicofísicos de mensuração visual, seria possível realçar essa diferença. ${ }^{\left({ }^{15}\right)}$ A escolha da tabela de AV pelo Early Treatment of Diabetic Retinopathy Study (ETDRS) foi o principal propósito dessa comparação, devido à sua precisão. ${ }^{(16)}$

Como forma também de aprimorar ainda mais o sentido de comparações entre as tabelas de AV neste estudo, foi proposto um método pouco utilizado na literatura, consistindo na cronometragem do tempo de leitura para perto por meio de cartões padronizados da tabela de Jaeger..$^{(17-21)}$

O objetivo deste estudo é comparar a acurácia entre a AV com melhor correção óptica (AVCC) mensurada pela tabela de Snellen em pacientes com RD e AVCC menor ou igual a 20/200 em ambos os olhos, com a mensuração pela tabela ETDRS, além de avaliar o tempo de leitura com tabela de leitura de Jaeger.

\section{MÉTODOS}

Estudo observacional e transversal realizado no Serviço de Oftalmologia do Hospital Universitário Antônio Pedro da Universidade Federal Fluminense (UFF), entre janeiro e dezembro de 2019. O presente estudo faz parte do plano de ensino de Iniciação Científica da Faculdade de Medicina da UFF, com aprovação do Comitê de Ética e Pesquisa (CEP) desta faculdade sob inscrição CAAE 79884817.2.0000.5243, seguindo os parâmetros da Declaração de Helsinki para pesquisa médica.

\section{Critérios de inclusão}

Foram incluídos pacientes com RD acompanhados no Ambulatório de Retina e Vítreo do Serviço de Oftalmologia do hospital, com correção óptica bilateral de 20/200 a 20/400 segundo a tabela de Snellen.

O critério de bilateralidade da condição da AV foi utilizado para homogeneizar a amostra coletada e não permitir que distorções visuais, como metamorfopsia, pudessem influenciar nos testes de leitura de perto, mensurados sob condições de binoculares.

Para prevenir viés referente à etiologia da baixa AV por opacidade de meios ópticos, comum em pacientes diabéticos, optou-se por selecionar pacientes pseudofácicos para este estudo.

\section{Critérios de exclusão}

Foram excluídos do estudo pacientes com importante opacidade de meios ópticos que não se conseguisse observar o fundus ocular e classificar a RD, além de AV imensurável aos testes psicofísicos. Da mesma forma, excluíram-se pacientes com campo visual tubular ou glaucoma avançado.

\section{Condutas e instrumentos da pesquisa}

Os pacientes foram avaliados no Ambulatório de Retina e Vítreo mensurando-se a AV com correção à distância de $5 \mathrm{~m}$ pela tabela de Snellen (iluminada; Xenônio; São Paulo, Brasil) e pela tabela ETDRS (iluminada; Xenônio; São Paulo, Brasil). Foi realizada, a seguir, a mensuração do tempo de leitura (em segundos) com o uso da tabela de Jaeger para perto a $33 \mathrm{~cm}$.

Após a avaliação da AV, sob dilatação pupilar com colírios midriáticos, realizou-se a classificação da RD em não proliferativa (RDNP) e RD proliferativa (RDP), sob avaliação à lâmpada de fenda (SL40; Zeiss; Jena, Alemanha) e uso de lentes panfundoscópicas (78 Dioptrias; Volk; Ohio, Estados Unidos). 


\section{Recursos simples de visão subnormal}

Para os pacientes especiais que não atingiram leitura mínima de Jaeger seis (J6), foram utilizados recursos simples, como adição refrativa igual ou acima de quatro dioptrias e lupas de magnificação manual de 4X de aumento. Esses casos foram descritos particularmente nos resultados do estudo como J6+, caso esse nível de leitura fosse atingido.

\section{Descrição dos testes psicofísicos}

A tabela de Snellen para exame da AV foi usada para caracterizar o ingresso no estudo segundo os critérios de inclusão.

A mensuração da AV foi realizada com a tabela ETDRS, para a avaliação comparativa com os valores da tabela de Snellen. A tabela ETDRS consiste em um método de leitura com letras em ordem decrescente, com contagem de linhas e letras.

Constituíram considerações especiais em relação à tabela de Jaeger para perto com cartão de leitura: ter pelo menos três linhas de palavras escritas no cartão em cada nível do grau da AV para perto, de modo que um indivíduo com AV normal com correção (20/20 ambos olhos) levasse pelo menos sete segundos cronometrados para ler a sentença de palavras do nível J1. Pacientes sem condições de leitura para perto, mesmo após utilizados todos os recursos ópticos, foram denominados sem leitura. Somente a primeira leitura do cartão foi considerada como passível de aceite pelo teste, de forma a não incentivar decorar palavras. Foi utilizada uma sala de leitura com condições padronizadas de iluminação com luminária de LED para avaliação de todos os casos pesquisados.

\section{Variáveis pesquisadas}

A variável principal foi a AV com a tabela de Snellen (valores menores do que 20/200 e igual a 20/200). As variáveis secundárias foram: tempo de leitura para perto com a tabela de Jaeger (cronometragem em segundos), AV com a tabela de leitura para perto de Jaeger (níveis J1, J2, J3, J4, J5 e J6; além de +J6 quando utilizar recursos simples de visão subnormal), AV para distância com a tabela ETDRS, classificação da RD (RDNP ou RDP), idade (média e desvio-padrão) e sexo (masculino ou feminino). A RDNP foi moderada, severa e muito severa e a RDP, moderada, alto risco e avançada.

\section{Formação de grupos de pesquisa}

Utilizando a tabela Snellen, o Grupo I foi formado por pacientes com AV igual a 20/200 (com subgrupos de RDNP e RDP) e o Grupo II por aqueles com AV menor que 20/200 (com subgrupos de RDNP e RDP). Para melhor análise de dados entre Tabelas de AV de Snellen, ETDRS e Jaeger se utilizará os níveis de maior que 20/200, 20/200, maior que 20/400, e 20/400 para comparações entre estas tabelas.

\section{Análise estatística}

Tabelas de contingência foram utilizadas pra dispor os dados da pesquisa e realizar inferências estatísticas. A análise estatística realizada neste estudo usou os testes $t$ de Student para avaliar as variáveis contínuas e intervalares; Kruskal-Wallis, para as variáveis ordinais e o qui-quadrado, para as variáveis nominais e tabulares. O nível de significância estatística do estudo é de $5 \%(\mathrm{p}<0,05)$

\section{RESULTADOS}

Após a aplicação dos critérios de inclusão e exclusão, foram incluídos no estudo 62 pacientes diabéticos (124 olhos): 33 pacientes com RDNP (dez classificados como moderados, 15 como severos e oito como muito severos) e 29 pacientes com RDP (13 classificados como moderados, dez como alto risco e seis com avançados). Da amostragem total pesquisada, 18 pacientes foram submetidos a tratamento prévio com fotocoagulação a laser.

Não houve sinais de hemorragia vítrea e nem de descolamento de retina tracional instável em atividade nos casos de RDP nos pacientes da amostragem pesquisada. Vinte e um pacientes com RDNP estavam em tratamento para edema macular diabético com terapia antiangiogênica. Não houve pacientes em tratamento com agentes antiangiogênicos entre os pacientes com RDP. Os dados gerais da pesquisa estão relatados na tabela 1.

Tabela 1. Dados gerais da amostragem pesquisada

\begin{tabular}{|c|c|c|c|c|c|c|}
\hline \multirow{2}{*}{$\begin{array}{l}\text { Dados } \\
\text { Demográficos }\end{array}$} & \multirow{2}{*}{ n (\%) } & \multirow{2}{*}{$\begin{array}{l}\text { Sexo } \\
\text { (M/F) }\end{array}$} & \multirow{2}{*}{$\begin{array}{c}\text { Idade } \\
\text { (média } \pm \text { DP) em } \\
\text { anos }\end{array}$} & \multicolumn{2}{|c|}{$\begin{array}{c}\text { Acuidade visual } \\
\text { (Snellen) } \\
\text { n (\%) }\end{array}$} & \multirow{2}{*}{$\begin{array}{l}\text { Valor } \\
\text { de } p^{*}\end{array}$} \\
\hline & & & & $\begin{array}{c}\text { AV } \\
20 / 200\end{array}$ & $\underset{20 / 400}{A V}$ & \\
\hline RDNP & $\begin{array}{c}33 \\
(52,4 \%)\end{array}$ & $18 / 15$ & $47,8 \pm 11,4^{+}$ & $\begin{array}{c}19 \\
(30,6 \%)\end{array}$ & $\begin{array}{c}14 \\
(22,5 \%)\end{array}$ & \multirow{2}{*}{0,04} \\
\hline RDP & $\begin{array}{c}29 \\
(47,6 \%)\end{array}$ & $9 / 20$ & $55,5 \pm 18,4^{\dagger}$ & $\begin{array}{c}13 \\
(20,9 \%)\end{array}$ & $\begin{array}{c}16 \\
(25,8 \%)\end{array}$ & \\
\hline
\end{tabular}

Em relação às variáveis demográficas, somente a idade obteve significância estatística, quando se compararam os grupos de pacientes com RDNP com os de pacientes com RDP $(\mathrm{p}=0,04)$. Embora esse dado obtenha uma significância estatística, essa inferência não foi estimada como expressiva na comparação da amostragem, uma vez que já era esperado esse tipo de resultado por outros estudos dessa morbidade ocular..$^{(7-9)}$ 
Os resultados da comparação da AV aferida com a tabela de Snellen e a ETDRS estão expostos na tabela 2. Não se encontraram pacientes com AV menor do que 20/400 com correção óptica neste estudo.

Tabela 2. Dados comparativos de mensuração da acuidade visual com correção obtidos com o emprego das tabelas Snellen e ETDRS

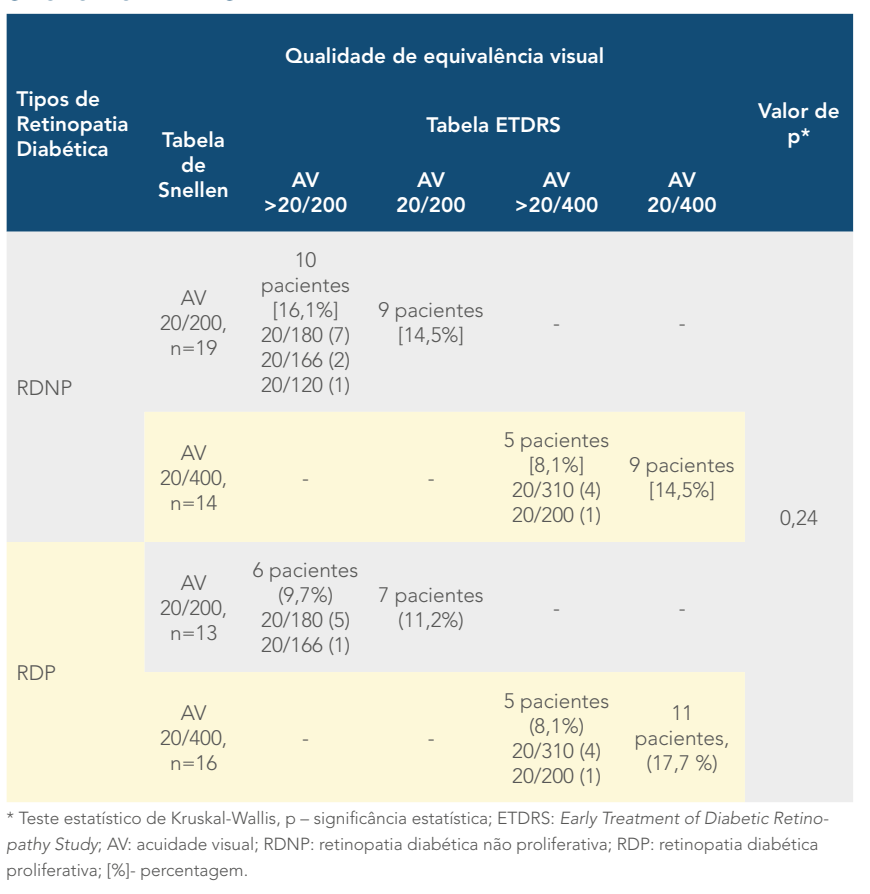

A tabela 2 mostra resultados de variabilidade de valores da AV dos pacientes aferida com a tabela ETDRS em relação à AV medida com a tabela de Snellen $(\leq 20 / 200)$, embora estes dados tabulares não tenham apresentado significância estatística.

O teste de AV aferido pela tabela ETDRS revelou 25,8\% de pacientes com AV maior que 20/200. Os pacientes diabéticos com RD e AV maior que 20/400 representaram $16,2 \%$ da amostra total.

Quando se compara os valores da AV avaliados pela tabela de Snellen com os obtidos com a tabela ETDRS, não houve significância estatística $(\mathrm{p}=0,24)$.

A comparação dos dados da AV (valores da tabela de Snellen versus tabela ETDRS versus valores da tabela de Jaeger versus tempo de leitura para perto) demonstrou significância estatística $(\mathrm{p}=0,04)$. Estes dados estão expostos na tabela 3 .

Os pacientes avaliados com índices de uso de recursos simples de visão subnormal somaram 31 casos (50\% da amostra) e casos sem leitura obtida pela tabela de Jaeger foram sete pacientes (11,2\% da amostra), predominantemente entre pacientes diabéticos com RDP.
Tabela 3. Dados da qualidade de equivalência visual referentes a comparações entre tabela de Snellen, tabela ETDRS e tabela de Jaeger para teste de velocidade de leitura de perto
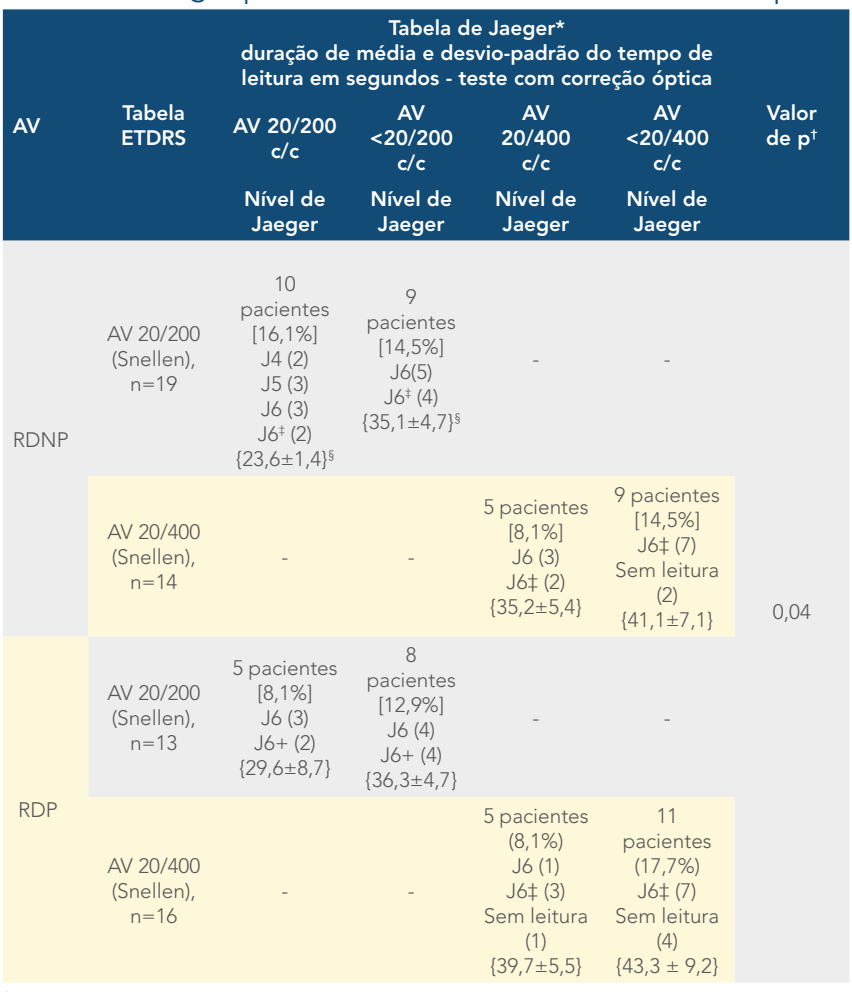

\{\} - Cronometragem em segundos, [\%] - porcentagem de amostras, † teste estatístico de t de Student; ‡ Uso de recursos de visão subnormal; ${ }^{5}$ valores com significância estatística; $(n)$ - número de pacientes em subgrupos. AV: acuidade visual; ETDRS: Early Treatment of Diabetic Retinopathy Study; cc: com correção óptica, RDNP: retinopatia diabética não proliferativa; RDP: retinopatia diabética proliferativa

\section{DISCUSSÃO}

Neste estudo, o critério de bilateralidade e de seleção de pacientes pseudofácicos, embora servisse como elemento de homogeneização da amostra, também contribuiu para um prolongamento do tempo de coleta de dados.

Comumente se sabe do ensejo do hábito de se utilizar a tabela de Snellen, devido à praticidade e à facilidade de aprendizado de uso. Porém, optou-se por comparar a tabela de Snellen com a tabela ETDRS, embora já se conhecessem as vantagens do uso de uma sobre a outra em razão da precisão do resultado. ${ }^{(13,15,17,1,18,22,23)}$ Entretanto, focalizando nos optotipos de 20/200 e 20/400 da tabela de Snellen com propósito de analisá-los sob conceitos de cegueira legal, desde a promulgação da portaria 3.128, em 2008, pelo Ministério da Saúde, esta resolução serve de diretriz legal para nortear as diferenças entre visão subnormal e cegueira legal, como também de cegueira total ou amaurose. Evidentemente, este estudo aborda apenas o lado de AVCC, pelo aspecto de bilateralidade, não contemplando avaliações sobre campo visual, conceito intrínseco ligado à cegueira legal..10-12)

Artigos publicados abordando as vantagens e desvantagens entre as tabelas de AV são discutidos na literatura, porém sem ressaltar o aspecto destas vantagens sobre os 
critérios de cegueira legal. ${ }^{(14,17-20)} \mathrm{O}$ presente estudo revela que $25,8 \%$ da amostra apresentou AV >20/200 pela tabela ETDRS, sendo a AV pela tabela de Snellen de 20/200, ou seja, caracterizada como cegueira legal. Embora esses dados tabulares não sejam considerados de estatística significativa, revelam que esta possibilidade existe.

Mesmo entre pacientes diabéticos com AV pior de que 20/400 pela tabela de Snellen, dez pacientes apresentaram AV menor que 20/400 pela tabela de ETDRS $(16,2 \%$ da amostra).

O próprio teste de tempo de leitura para perto com tabela de Jaeger revelou média e desvio-padrão estatisticamente significativos para o grupo estimado como maior de 20/200 de AV com correção, coincidindo em 82\% com o mesmo grupo pesquisado com a tabela ETDRS para a distância com a amostragem de maior de 20/200. Essa maior facilidade de leitura é realmente mais compatível com pacientes com algum tipo de deficiência visual, mas está abaixo dos índices de cegueira legal. Esse conceito é pouco explorado na literatura. ${ }^{(20,24)}$

Como critério de mais rígido aspecto de comparação na literatura, existe para o ingresso nas Forças Armadas, a necessidade de uma boa capacidade visual, pois é imprescindível na manipulação de equipamentos de alta precisão e instrumentos de alta periculosidade. Deve-se se ter maior precisão para aferir a AV, chegando a se levantar a hipótese de uma metodologia mais rigorosa do que a tabela de Snellen, porém o nível de exigência, na prática atualmente, ainda é o valor de AV da tabela de Snellen (20/20).(24-26)

As limitações deste estudo estão primeiramente relacionadas com pequeno número da amostra total, que poderia ser mais estatisticamente expressiva caso houvesse um número maior de participantes. A exclusão de participantes não pseudofácicos corroborou também para um menor tamanho da amostra, além do aspecto da bilateralidade na metodologia. Embora se tenha observado o critério de bilateralidade para o conceito de deficiência visual, o presente estudo não considerou o critério de avaliação de campo visual, sendo apenas excluídos os casos extremos de glaucoma.

\section{CONCLUSÃO}

A tabela de Snellen possui maior facilidade de entendimento para seu uso prático fora da oftalmologia e mesmo fora da área médica entre leigos, porém, ao se assumirem aspectos legais para condição laborativa para cegueira legal, tabelas como Bailey-Lovie ou ETDRS deveriam ser mais utilizadas, por apresentarem maior precisão na avaliação da capacidade visual.

\section{REFERÊNCIAS}

1. Bicas HE. Acuidade visual. Medidas e notações. Arq Bras Oftalmol. 2002;65(3):375-84.

2. Leal Barros DN. Sociedade Brasileira de Visão Subnormal. Classes de Acuidades Visual Classificação ICD-9-CM(WHO/ICO): Acuidade Visual Snellen. [citado 2020 Abr 16]. Disponível em http://www.cbo.com.br/ subnorma/conceito.htm

3. Westheimer G. Scaling of visual acuity measurements. Arch Ophthalmol.1979;97(2):327-30.

4. Pereira CF, Costa R, Del Ciampo LA, Ferraz I. Triagem de acuidade visual reduzida em uma unidade de Atenção Primária à Saúde. Rev Bras Oftalmol. 2019;78(4):250-4

5. Princípios da Oftalmologia - Avaliação e consulta oftalmológica. In - Liga de Oftalmologia. Disponível em: http://ligadeoftalmo.ufc.br/arquivos/... avaliacao_oftalmologica.pdf. Acesso em 12/09/2020.

6. Klein BE. Overview of epidemiologic studies of diabetic retinopathy. Ophthalmic Epidemiol. 2007;14(4):179-83.

7. Shaw JE, Sicree RA, Zimmet PZ. Global estimates of the prevalence of diabetes for 2010 and 2030. Diabetes Res Clin Pract. 2010;87(1):4-14.

8. Turco PD, Connolly J, McCabe P, Glynn RJ. Assessment of functional vision performance: a new test for low vision patients. Ophthalmic Epidemiol. 1994;1(1):15-25

9. International Diabetes Federation. Diabetes Atlas. Prevalence estimates of Diabetes Mellitus (DM). Available at http://www.diabetesatlas.comhttp:// www.diabetesatlas.com/content/prevalence-estimates-2018/content/ prevalence-estimates-2018

10. World Health Organization (WHO). Blindness and visual impairement. Geneve: WHO; c2021. Avaliable from: https://www.who.int/news-room/ fact-sheets/detail/blindness-and-visual-impairment

11. Brasil. Ministério da Saúde. Gabinete do Ministro. Portaria no. 3.128, de 24 de dezembro de 2008. Define que as Redes Estaduais de Atenção à Pessoa com Deficiência Visual sejam compostas por ações na atenção básica e Serviços de Reabilitação Visual [Internet]. Brasília, DF: Diário Oficial da União, 2008 [citado 2021 Mai 26]. Disponível em: http://bvsms. saude.gov.br/bvs/saudelegis/gm/2008/prt3128_24_12_2008.html

12. World Health Organization (WHO). The prevention of blindness : report of a WHO Study Group [meeting held in Geneva from 6 to 10 November 1972] [Internet]. Genève: WHO; 1973 [cited 2021 May 26]. Available from: https://apps.who.int/iris/handle/10665/38222

13. Código CID-10 - Subtópico de conceitos de visão subnormal . Disponível em: https://www.medicinanet.com.br/cid10/1689/h54_cegueira_e_visao_ subnormal.htm

14. Costa $A L$, Santos VR. Da visão à cidadania: tipos de tabela avaliação funcional da leitura na educação especial. Rev Bras Oftalmol. 2018;77(5):296-302.

15. Rosser DA, Cousens SN, Murdoch IE, Fitzke FW, Laidlaw DA. How sensitive to clinical change are ETDRS logMAR visual acuity measurements? Invest Ophthalmol Vis Sci. 2003;44(8):3278-81.

16. França VC, Costa MF. Diferenças de acuidade visual por três métodos psicofísicos na Tabela ETDRS. Neurociências. 2009;5(3):33-8.

17. Kusuhara S, Fukushima Y, Ogura S, Inoue N, Uemura A. Pathophysiology of diabetic retinopathy: the old and the new. Diabetes Metab J. 2018;42(5):364-76.

18. Sloan LL. New test charts for the measurement of visual acuity at far and near distances. Am J Ophthalmol. 1959;48:807-13.

19. Bailey IL, Lovie JE. New design principles for visual acuity letter charts. AmJ Optom Physiol Opt. 1976;53(11):740-5.

20. Schwiegerling J. Theoretical limits to visual performance. Surv Ophthalmol 2000;45(2):139-46.

21. Lim LA, Frost NA, Powell RJ, Hewson P. Comparison of the ETDRS logMAR, compact reduced logMAR and Snellen charts in routine clinical practice. Eye (Lond). 2010;24(4):673-7.

22. Idil A, Caliskan D, Ocaktan E. The prevalence of blindness and low vision in older onset diabetes mellitus and associated factors: a community-based study. Eur J Ophthalmol. 2004;14(4):298-305. 
23. Ferris FF, Kassoff A, Bresnick GH, Bailey I. New Visual Acuity Charts for Clinical Research. Am J Ophthalmol. 1982;94(1):91-6.

24. Weaver JL, McAlister WH. Vision readiness of the reserve forces of the U.S. Army. Mil Med. 2001;166(1):64-6
25. Erneston AG, Ricks MR, Tate TJ, Ana RS. Vision readiness in the United States Air Force revisited. Mil Med. 1996;161(1):27-8.

26. Erneston AG, Murchland MR. Questioning vision readiness in the aviation community of the United States Air Force. Mil Med. 1994;159(6):432-4. 